\title{
Parallel Interference Cancellation for MIMO Radar Receiver Ying-Hua Tian ${ }^{1, a}{ }^{*}$, Song Chen ${ }^{1, b}$,Sheng Wang ${ }^{1, c}$ and Xin-Peng Chen ${ }^{2, d}$ \\ ${ }^{1}$ Zhengzhou Institute of Information Science and Technology, Zhengzhou, China \\ ${ }^{2}$ Institute of Command Automation PLA University of Science and Technology Nanjing,China atian.ying.hua@foxmail.com, ${ }^{b}$ wirelessmancs@163.com, cwsptrieu@126.com, ${ }^{d}$ cxphit@163.com
}

\section{Keywords: MIMO Radar; Interference Cancellation; Pulse Compression}

\begin{abstract}
MIMO radar systemstransmit different waveforms simultaneously to obtain multiple observation channels towards target, which also brings in interference between those signals. This paper introduces the communication technique denoted as parallel interference cancellation (PIC)into MIMO radar receiver. For the return signals from target, the proposed method cancels the interference by using the prior knowledge of transmit signals. PIC is found to yield substantial performance improvement in MIMO radar system compared with standard match filters usually used. Finally, simulation results demonstrate the effectiveness of the proposed method.
\end{abstract}

\section{Introduction}

Multiple-Input-Multiple-Output (MIMO) radar systems[1,2] are getting attention nowadays for their potential advantages, such as extended array aperture, better estimation resolution and increased spatial diversity. It transmits different waveforms to illuminate the target from different antennas simultaneously. Therefore, each MIMO radar receiver receives a set of reflected signals overlapped. Theoretically, transmitted waveforms can be designed to be orthogonal from each other so that we can use match filter to extract each waveform as well as the target parameter. However, due to the limited waveform length there are no fully orthogonal waveforms in fact, which lead to interference between multiple transmitted signals.

Some efforts are made to solve the problem by designing the waveform set with good cross-correlation $[3,4]$. However, those waveforms are difficult to findand the cross-correlation of waveform still exists, which influences the receiver performance. Others attempt to suppress this kind of interference by design adaptive receiver filter[5,6].But those approaches demand computational cost of $O\left(K^{3}\right)$ where $K$ is the length of filter, and matrix inversion operation which is usually unstable.

Considering that the notion of MIMO radar was introduced from MIMO communication, and the way we use orthogonal waveforms set to achieve multiple measurement channels towards target is similar to themulti-user communication techniques, especially the code-division multiple-access (CDMA) system, it is reasonable to introduce methods used in communication area to solve the multiple channel interference in MIMO radar. In CDMA communication, several users share the same spectrum to transfer signals and each is identified by its distinct transmitted code.It also brings in the multiple-access interference (MAI) problem that is similar to the interference in MIMO radar[7]. One of the most used approach to suppress the MAIin CDMA communication is parallel interference cancellation (PIC), which can improve the receiver performance by using the prior knowledge of interference.[8]

In this paper,weproposea newMIMO radar receiver based on PICto cancel the multi-channelinterference in MIMO radar and then numerical simulation are applied to verify the capability of range sidelobe reduction.The proposed method can cancel the multi-channel interference effectively and demand less computational cost than that in [5]. 


\section{MIMO Radar Signal Model}

The structure of MIMO radar used in this paper is illustrated in Fig.1. The transmit antennas transmit $\mathrm{M}$ orthogonal signals, which are reflected by the target and then received by the receiver antennas. For simplicity, we ignore Doppler effects and consider the RCS is constant during a pulse period. The transmitted baseband signals are denoted by $S_{m} \in C^{1 \times K}$, when $\mathrm{m}$ denotes the $m$ th transmitter. Assume that there is a target with direction of departures (DODs) $\theta$ and direction of arrivals (DOAs) $\varphi$. The signal received by the nth receiver can be described by the expression

$$
\begin{aligned}
& \vec{x}_{n}=\sum_{i=1}^{M} \alpha_{i n} \vec{s}_{i}+\vec{w}_{n}(1) \\
& \alpha_{i n}=a_{r}(\theta) a_{t}^{T}(\varphi) e
\end{aligned}
$$

Where $e \in C$ denotes the RCS of the target. $a_{r}(\theta) \in \mathrm{C}^{1 \times N}$ is the steering vector of receiver array and $a_{t}(\varphi) \in \mathrm{C}^{1 \times M}$ is the transmit array steering vector. $\alpha_{i n} \in C$ denotes the gain of observation channel between ith transmit antenna and $n$th receiver antenna. $\vec{w}_{n} \in C^{1 \times K}$ denotes noise in the receiver. We consider a jammer free model in which $\vec{w}_{n}$ is an independent Gaussian noise and $\vec{w} \vec{w}^{H}=\sigma^{2}$.

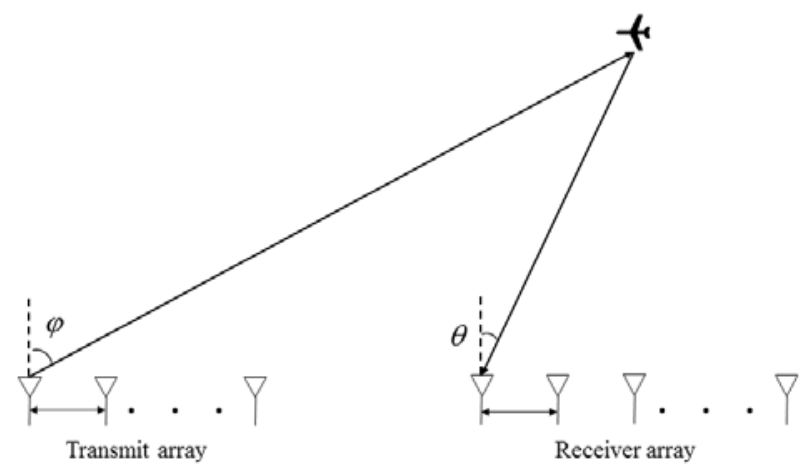

Fig.1. Basic MIMO radar scenario.

\section{Parallel Interference Cancellation}

In this section, the parallel interference cancellation method was derived to cancel the effects of the cross-correlation of the transmit waveform.

Matching the received signalwith $s_{m}^{H}$, we obtain

$$
\vec{y}_{m n}=\vec{x}_{n} \vec{s}_{m}^{H}=\alpha_{m n} \vec{s}_{m} \vec{s}_{m}^{H}+\sum_{i \neq m} \alpha_{i n} \vec{s}_{i} \vec{s}_{m}^{H}+\vec{w}_{n} \vec{s}_{m}^{H}
$$

Where $\frac{1}{K} \sum_{i \neq m} \alpha_{i n} \vec{s}_{i} \vec{s}_{m}^{H}$ is just the interference as a result of non-zero cross-correlation between transmit waveforms.

It is clear that if we remove the second term from right side of the Eq. 3, the interference would be cancelled.It can be done by simply subtracting the estimation of this term from the equation. Furthermore, if we obtain the estimation of $\alpha_{i n}$ where $(i \neq m)$, the interference could be eliminated even before the match filter as shown in Eq. 3. 


$$
\begin{aligned}
\vec{y}_{\text {ICmn }} & =\alpha_{m n} \vec{s}_{m} \vec{s}_{m}^{H}+\sum_{i \neq m} \alpha_{i n} \vec{s}_{i} \vec{s}_{m}^{H}+\vec{w}_{n} \vec{s}_{m}^{H}-\sum_{i \neq m} \hat{\alpha}_{i n} \vec{s}_{i} \vec{s}_{m}^{H} \\
& =\left(\vec{x}_{n}-\sum_{i \neq m} \hat{\alpha}_{i n} \vec{s}_{i}\right) \vec{s}_{m}^{H} \\
& =\vec{x}_{I C m n} \vec{s}_{m}^{H}
\end{aligned}
$$

Where

$$
\vec{x}_{I C m n}=\vec{x}_{n}-\sum_{i \neq m} \hat{\alpha}_{i n} \vec{s}_{i}
$$

In Eq. 4, $\vec{y}_{\text {ICmn }}$ denotes the output of match filter after the interference cancellation method and $\hat{\alpha}_{i n}$ denotes the initial estimateof $\alpha_{i n}$, which can be obtained by applying a match filter bank.

$$
\hat{\alpha}_{i n}=\vec{X}_{n} \vec{s}_{i} / \vec{s}_{i} \vec{S}_{i}^{H}
$$

The whole signal flow of parallel interference cancellation is illustrated in Fig. 2in which all of the match filter bank are applied to estimate the initial gain and range profiles firstly. The estimates are then employed to refine the output of each match filter at next stage.This process of refining the output of match filter and then re-estimating the target profiles can be repeated for number of stages to obtain an ideal output.

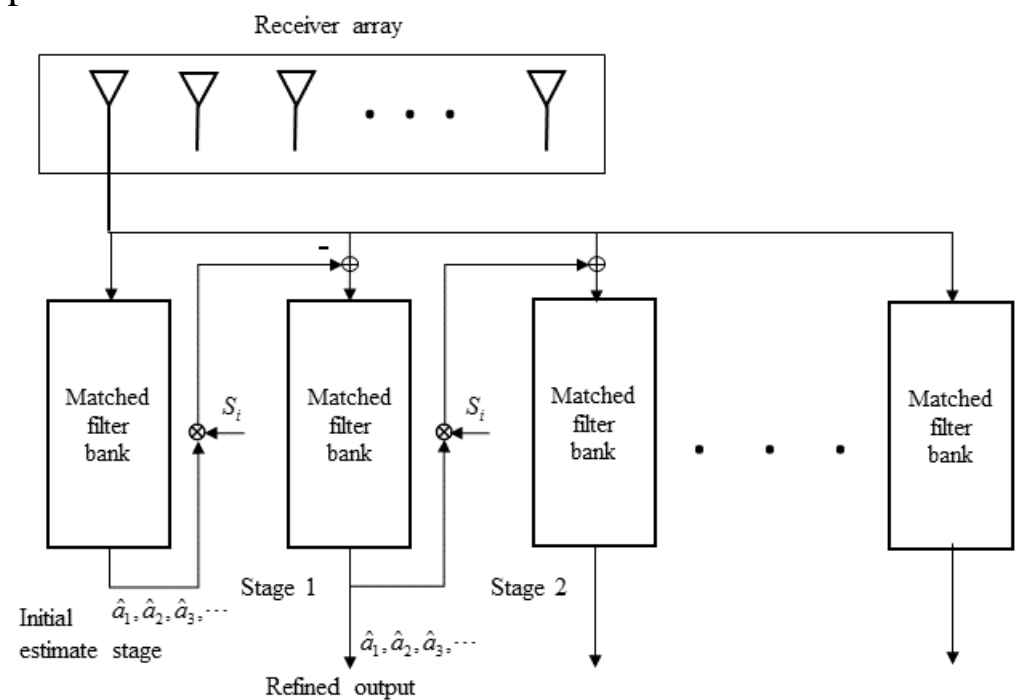

Fig.2. Signal Flow of Parallel Interference Cancellation.

At each stage of the PIC process,the input signal is subtracted by the prior estimate of interference and a set of standard match filter bank is applied to estimate channel gain. The total complexity computation, most of which comes from the match filter bank, is proportional to $K^{2}$ when the adaptive filtering method demand computational cost of $O\left(K^{3}\right)$ [5,9].The method proposed is also more stable than adaptive filter methods for the lack of matrix inversion operation.

\section{Simulation Results}

Considering a MIMO radar system consisting of eight transmit antennas and eight receiver antennas, the space of transmitting element and receive element is half-wavelength. Furthermore, a set of polyphase codes with $\mathrm{L}=128$ and $\mathrm{M}=8$ designed by Gold sequences generator is applied. Each element concurrently transmits a different waveform,the auto-correlations and cross-correlation of which are depicted in Fig. 3. 

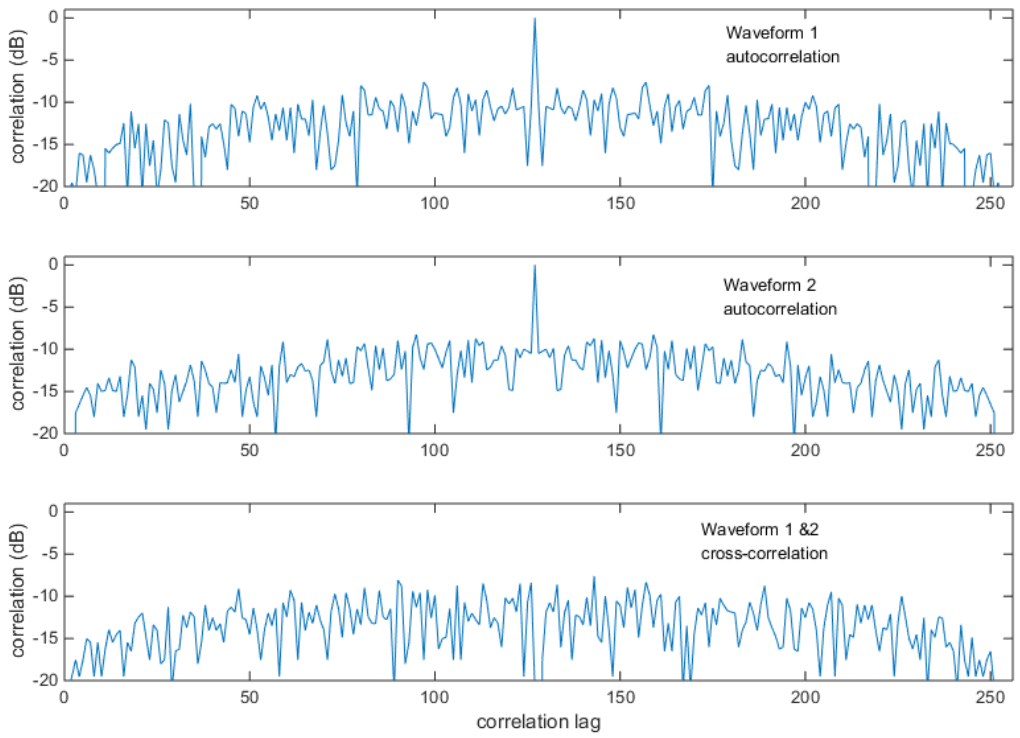

Fig.3. Correlations of transmitted waveforms.

A still target is assumed to be illuminated by the MIMO radar's main beam. The SNR level of received signal is set to $5 \mathrm{~dB}$. To demonstrate the performance of the PIC receiver, we compare its performance with that of match filtering andthe ground truth of the waveform's autocorrelation.

The results for this scenario are illustrated in Fig. 4, in which the black line represents the ground truth of respective composite range profiles. For the illustrative purposes, we apply the match filter and 1-stage PIC receiver to the received signal. As expected, the match filter estimate is rather poor due to the presence of other waveforms transmitted at the same time, yielding a filter output with a $-5 \mathrm{~dB}$ peak sidelobe level. However the PIC approach outperforms the match filtering with a-14dB peak sidelobe level [10].

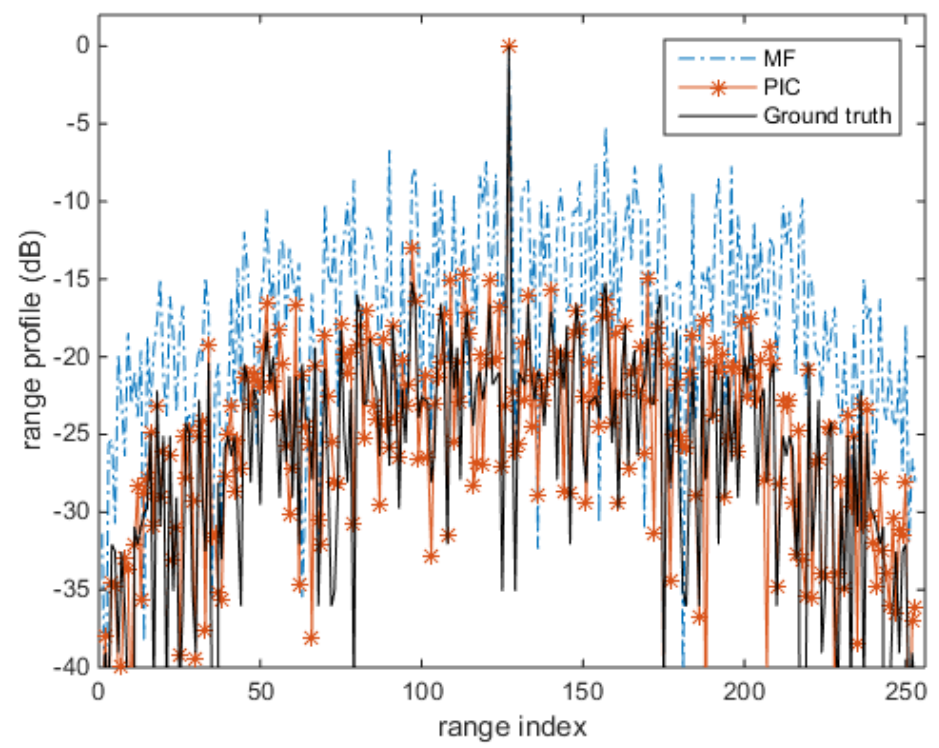

Fig.4. composite range profile of target $(\mathrm{M}=8)$.

Fig. 5 shows that for a scenario with $\mathrm{M}=20$ transmit antennas, the PIC receiver outperforms significantly the standard match filter. The peak sidelobe level of PIC receiver is -13dB when the correlated peak of match filter is severely submerged in the sidelobe. 


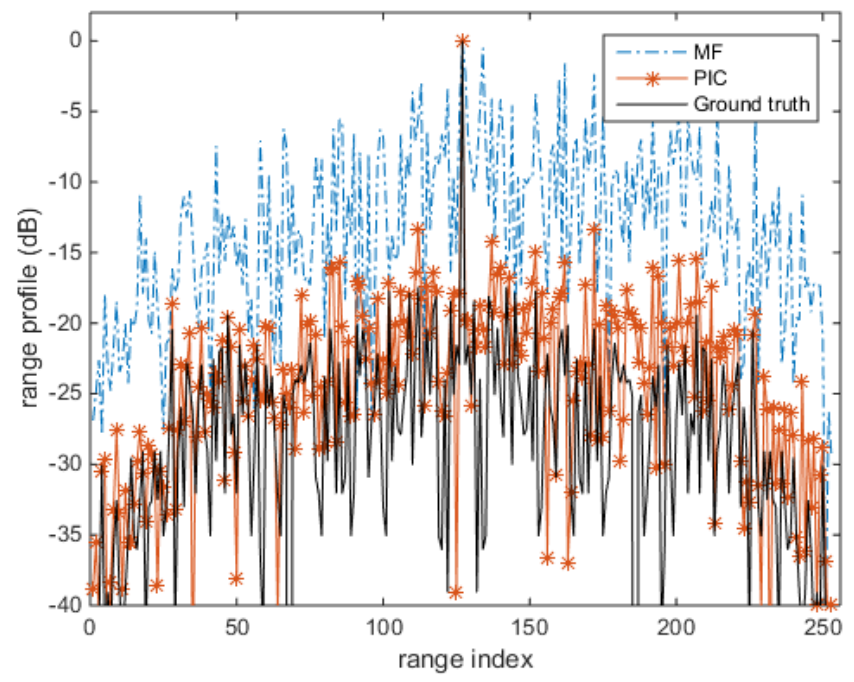

Fig.5. composite range profile of target $(\mathrm{M}=8)$.

\section{Conclusion}

Parallel interference cancellation has been introduced to improve MIMO radar receivers. It is shown thatthecomputationloadofthe proposed method is much lower than that of adaptive filter methods. The impact of interferences caused by the cross-correlation of transmit waveformscan be canceled effectively by the proposed method, especially in the MIMO radar systems that have a great many of transmit antennas or use a large set of waveforms.

\section{References}

[1] E. Fishler, A. Haimovich, R. Blum, D. Chizhik, L. Cimini, R. Valenzuela, MIMO radar: an idea whose time has come, in: Radar Conference, 2004. Proceedings of the IEEE, IEEE, 2004: pp. 71-78.

[2] J. Li, P. Stoica, MIMO radar with colocated antennas, Signal Processing Magazine, IEEE. 24 (2007) 106-114.

[3] H. Deng, Polyphase code design for orthogonal netted radar systems, Signal Processing, IEEE Transactions on. 52 (2004) 3126-3135.

[4] B. Liu, Z. He, J. Zeng, B. Liu, Polyphase orthogonal code design for MIMO radar systems, in: 2006 CIE International Conference on Radar, 2006: pp. 1-4.

[5] S.D. Blunt, K. Gerlach, Multistatic adaptive pulse compression, Aerospace and Electronic Systems, IEEE Transactions on. 42 (2006) 891-903.

[6] H.J. Blinchikoff, Range sidelobe reduction for the quadriphase codes, Aerospace and Electronic Systems, IEEE Transactions on. 32 (1996) 668-675.

[7] M.K. Varanasi, B. Aazhang, Multistage detection in asynchronous code-division multiple-access communications, Communications, IEEE Transactions on. 38 (1990) 509-519.

[8] S. Moshavi, E.G. Kanterakis, D.L. Schilling, Multistage linear receivers for DS-CDMA systems, International Journal of Wireless Information Networks. 3 (1996) 1-17.

[9] S.D. Blunt, K. Gerlach, Adaptive pulse compression via MMSE estimation, Aerospace andElectronic Systems, IEEE Transactions on. 42 (2006) 572-584.

[10]J. Jedwab, K. Yoshida, The peak sidelobe level of families of binary sequences, Information Theory, IEEE Transactions on. 52 (2006) 2247-2254. 